\title{
Black Hole Evolution Traced Out with Loop Quantum Gravity
}

\author{
Loop quantum gravity - a theory that extends general relativity by quantizing \\ spacetime-predicts that black holes evolve into white holes.
}

\section{by Carlo Rovelli*}

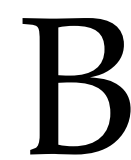

lack holes are remarkable entities. On the one hand, they have now become familiar astrophysical objects that have been observed in large numbers and in many ways: we have evidence of stellar-mass holes dancing around with a companion star, of gigantic holes at the center of galaxies pulling in spiraling disks of matter, and of black hole pairs merging in a spray of gravitational waves.

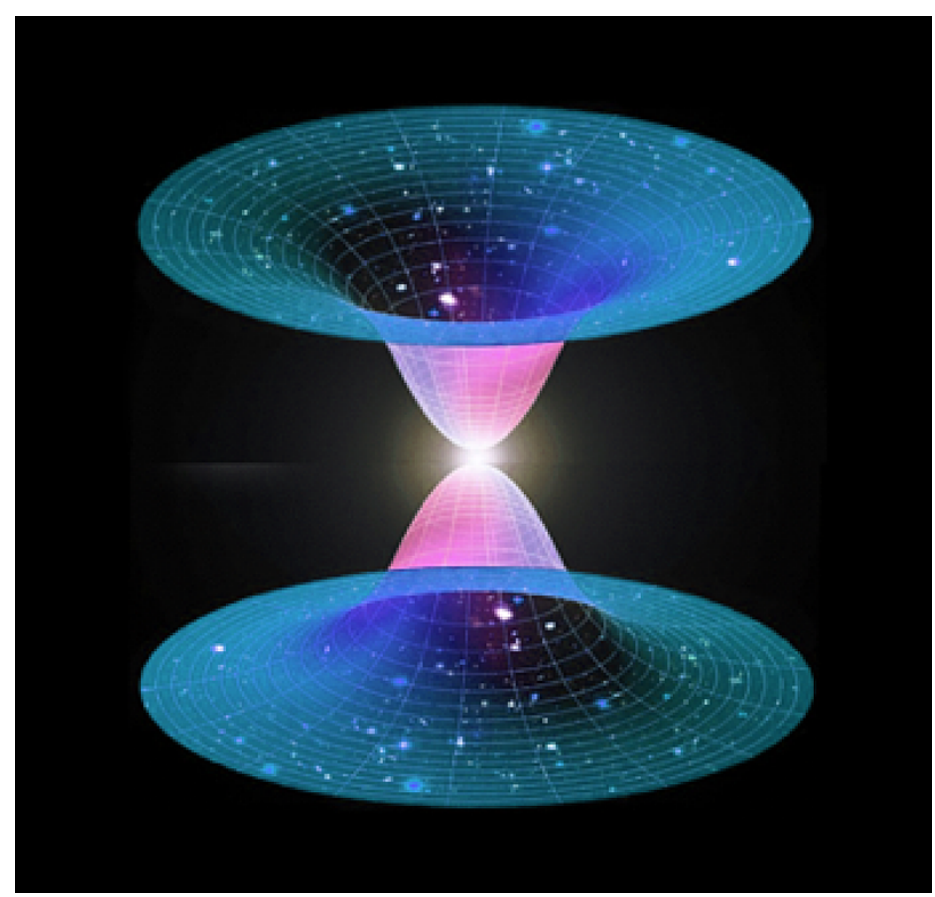

Figure 1: Artist rendering of the black-to-white-hole transition. Using loop quantum gravity, Ashtekar, Olmedo, and Singh predict that black holes evolve into white holes. (F. Vidotto/University of the Basque Country) *Center of Theoretical Physics, CNRS, Aix-Marseille University and
Toulon University, Marseille, France
All of this is beautifully accounted for by Einstein's centuryold theory of general relativity. Yet, on the other hand, black holes remain highly mysterious. We see matter falling into them, but we are in the dark about what happens to this matter when it reaches the center of the hole.

Abhay Ashtekar and Javier Olmedo at Pennsylvania State University in University Park and Parampreet Singh at Louisiana State University, Baton Rouge, have taken a step toward answering this question [1]. They have shown that loop quantum gravity - a candidate theory for providing a quantum-mechanical description of gravity-predicts that spacetime continues across the center of the hole into a new region that exists in the future and has the geometry of the interior of a white hole. A white hole is the time-reversed image of a black hole: in it, matter can only move outwards. The passage "across the center" into a future region is counterintuitive; it is possible thanks to the strong distortion of the spacetime geometry inside the hole that is allowed by general relativity. This result supports a hypothesis under investigation by numerous research groups: the future of all black holes may be to convert into a real white hole, from which the matter that has fallen inside can bounce out. However, existing theories have not been able to fully show a way for this bounce to happen. That loop quantum gravity manages to do it is an indication that this theory has ripened enough to tackle real-world situations.

The reason why we are in the dark about aspects of black hole physics is that quantum phenomena dominate at the center and in the future of these objects. Classical general relativity predicts that a black hole lives forever and that its center is a "singularity" where space and time end. These predictions are not realistic because they disregard quantum effects. To tackle these effects we need a quantum theory of gravity. We don't yet have consensus on such a theory, but we have candidates, some of which are now reaching the point of allowing actual calculations on the quantum behavior of black holes. Loop quantum gravity, which has a clean conceptual structure and a well-defined mathematical formulation based on representing the fabric of space as a spin network that evolves in time, is one such theory.

During the last few years, a number of research groups have applied loop theory to explore the evolution of black 


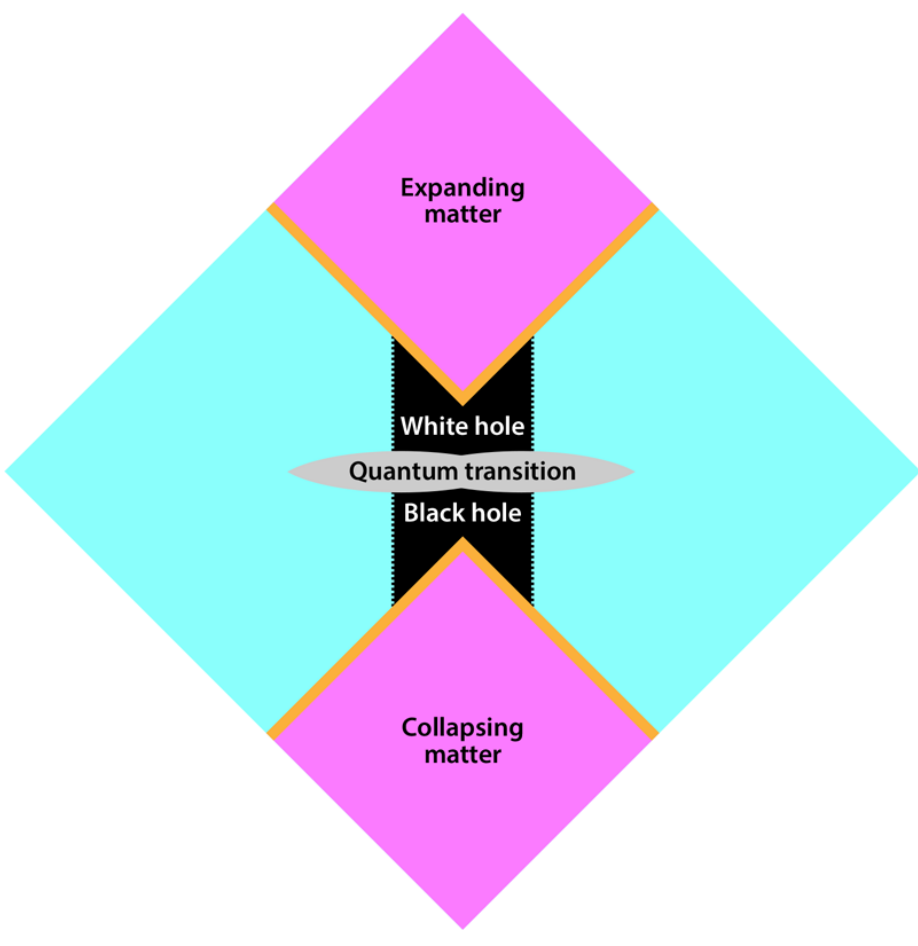

Figure 2: Diagram representing the spacetime evolution of a black hole into a white hole via a quantum transition. The vertical axis represents time; the horizontal axis represents distance from the center. (C. Rovelli/Aix-Marseille University; adapted by APS/Alan Stonebraker)

holes. These efforts are building a compelling picture based on a black-to-white-hole transition scenario (Fig. 1), which can be summarized as follows [2]. At the center of the black hole, space and time do not end in a singularity, but continue across a short transition region where the Einstein equations are violated by quantum effects. From this region, space and time emerge with the structure of a white hole interior, a possibility suggested in the 1930s by physicist John Lighton Synge [3]. As the hole's center evolves, its external surface, or "horizon," slowly shrinks because of the emission of radiation-a phenomenon first described by Stephen Hawking. This shrinkage continues until the horizon reaches the Planck size (the characteristic scale of quantum gravity) or earlier [4, 5], at which point a quantum transition ("quantum tunneling") happens at the horizon, turning it into the horizon of a white hole (Fig. 2). Thanks to the peculiar distorted relativistic geometry, the white hole interior born at the center joins the white horizon, completing the formation of the white hole.

Loosely speaking, the full phenomenon is analogous to the bouncing of a ball. A ball falls to the ground, bounces, and then moves up. The upward motion after the bounce is the time-reversed version of the falling ball. Similarly, a black hole "bounces" and emerges as its time-reversed ver- sion-a white hole. Collapsing matter does not disappear at the center: it bounces up through the white hole. Energy and information that fell into the black hole emerge from the white hole. The configuration where the compression is maximal, which separates the black hole from the white hole, is called a "Planck star." Because of the huge time distortion allowed by relativity, the time for the process to happen can be short (microseconds) when measured from inside the hole but long (billions of years) when measured from the outside. Black holes might be bouncing stars seen in extreme slow motion.

This is a compelling picture because it removes the singularity at a black hole's center and resolves the paradox of the apparent disappearance of energy and information into a black hole. Until now, this black-to-white-hole picture was not derived from an actual quantum theory of gravity; it was just conjectured-and implemented with ad hoc modifications to Einstein's general relativity equations. Ashtekar, Olmedo, and Singh have shown that a crucial ingredient of this scenario, the transition at the center, follows from a genuine quantum gravity theory, namely, loop theory. The result was obtained through an approximation of the full loop-quantum-gravity equations [6] — similar to the one employed in previous work aimed at resolving the big bang singularity [7].

It is important to note that the Ashtekar-Olmedo-Singh model addresses only the transition at the center of the hole. To complete the picture, we also need the calculation of the tunneling at the horizon [5]. Preliminary steps in this direction have been taken, but the problem is open. Its solution would lead to a complete understanding of the quantum physics of black holes.

It is not implausible that empirical observations could support this scenario. Models suggest that several observed astrophysical phenomena could be related to the black-towhite-hole transition [8]. Among these are fast radio bursts (FRBs) and certain high-energy cosmic rays. Both could be produced by matter and photons that were trapped in black holes produced in the early Universe and liberated by the black-to-white-hole transition. For the moment, however, the astrophysical data are insufficient to determine whether the statistical properties of observed FRBs and cosmic rays confirm this hypothesis [8]. Another intriguing possibility is that small holes produced by the black-to-white-hole transition may be stable: in which case, these "remnants" could be a component of dark matter [9].

We are only beginning to understand the quantum physics of black holes, but in this still speculative field, the AshtekarOlmedo-Singh result gives us a welcome fixed point: loop gravity predicts that the interior of a black hole continues into a white hole. The importance of any progress in this field goes beyond understanding black holes. The center of a black hole is where our current theory of spacetime, as given by Einstein's general relativity, fails. Understanding 
the physics of this region would mean understanding quantum space and quantum time.

This research is published in Physical Review Letters and Physical Review D.

\section{REFERENCES}

[1] A. Ashtekar, J. Olmedo, and P. Singh, "Quantum transfiguration of Kruskal black holes," Phys. Rev. Lett. 121, 241301 (2018); A. Ashtekar, J. Olmedo, and P. Singh, "Quantum extension of the Kruskal spacetime," Phys. Rev. D 98, 126003 (2018).

[2] E. Bianchi, M. Christodoulou, F. D’Ambrosio, H. M. Haggard, and C. Rovelli, "White holes as remnants: A surprising scenario for the end of a black hole," Class. Quant. Grav. 35, 225003 (2018).

[3] J. L. Synge, "The gravitational field of a particle," Proc. Roy. Irish Acad. A 53, 83 (1950).

[4] C. Rovelli and F. Vidotto, "Planck stars," Int. J. Mod. Phys. D 23, 1442026 (2014).
[5] H. M. Haggard and C. Rovelli, "Quantum-gravity effects outside the horizon spark black to white hole tunneling," Phys. Rev. D 92, 104020 (2015).

[6] L. Modesto, "Black hole interior from loop quantum gravity," Adv. High Energy Phys. 2008, 459290 (2008).

[7] I. Agullo and P. Singh, "Loop quantum cosmology: A brief review," Loop Quantum Gravity, 100 Years of General Relativity Vol. 4, edited by A. Ashtekar and J. Pullin (World Scientific, Singapore, 2017).

[8] A. Barrau, B. Bolliet, F. Vidotto, and C. Weimer, "Phenomenology of bouncing black holes in quantum gravity: A closer look," J. Cosmol. Astropart. Phys. 2016, 022 (2016); A. Barrau, K. Martineau, and F. Moulin, "Status report on the phenomenology of black holes in loop quantum gravity: Evaporation, tunneling to white holes, dark matter and gravitational waves," Universe 4, 102 (2018).

[9] C. Rovelli and F. Vidotto, "Small black/white hole stability and dark matter," Universe 4, 127 (2018).

10.1103/Physics.11.127 